\title{
Risk factors associated with hemorrhagic stroke in patients supported with continuous flow- LVAD
}

\author{
Arshi $\mathrm{J}^{1}$, JavidUlla $\mathrm{K}^{2}$, Bansal $\mathrm{R}^{3}$, Islam $\mathbf{M}^{4}$, Narayanaswamy $\mathbf{M}^{5}$, Azeem Husain $\mathrm{A}^{6}$, Hafsa ${ }^{7}$, Kaleem $\mathrm{A}^{8}$ and
} Faisaluddin $\mathbf{M}^{1 *}$

${ }^{1}$ Department of Internal Medicine, Owaisi General Hospital \& Research Center, India

${ }^{2}$ Department of Medicine, Deccan College of Medical sciences, India

${ }^{3}$ Department of Medicine, Gian Sagar Medical College and Hospital, India

${ }^{4}$ Department of Medicine, Sir Salimullah Medical College, Mitford Hospital, India

${ }^{5}$ Department of Internal Medicine, Deccan College of Medical Sciences, India

${ }^{6}$ Department of Medicine, Deccan College of Medical sciences, India

${ }^{7}$ Department of Internal Medicine, Dr. PSIMS \& RF, India

${ }^{8}$ Department of Medicine, Nishtar Medical College, Pakistan

Submission: August 16, 2018; Published: August 27, 2018

*Corresponding author: Faisaluddin M, Department of Internal Medicine, Owaisi General Hospital \& Research Center, India, Tel: 9949182319; Email: mfaisal.uddin47@gmail.com

\begin{abstract}
Background: Long term LVAD support can result in serious complication, such as stroke, bleeding, and infections. In this study, we investigated the risk factors associated with stroke in patients who underwent LVAD placement.

Methods: We evaluated 2465 patients (mean age 61) who underwent implantation of HeartMate II (Thoratec Corp., Pleasanton, CA) or Heart Ware (Framingham, MA) at our center between March 2012 and July 2017.

Results: The mean patient age was 61 (SD, 7.5 years) with mean body mass index of 31 (SD, 5.9). The mean follow-up was 13.9 months (SD 9.45). During the study period, 2465 patients underwent continuous flow LVAD placement. Median follow-up was 9.79 months (range 0.02 to 34.96 months). Of all patients, $829(33.63 \%)$ had at least 1 stroke, with an incidence rate of 0.336 strokes per patient-year. A total of 356 (42.94\%) strokes were ischemic and 311 (37.522\%) were hemorrhagic. Patients with hemorrhagic stroke had worse survival than those with ischemic strokes (90-day survival: $28.3 \%$ vs. $71.7 \%$; p < 0.001). Of patients with a first stroke, $21 \%$ had a second stroke. Pre-implant predictors of stroke were female sex (Hazard Ratio [HR]: 1.25; 95\% confidence interval [CI]: 1.09 to 1.82; p 0.001), pre-implant systolic blood pressure (HR: 1.87; 95\% CI: 1.00 to 1.01; p = 0.001), heparin-induced thrombocytopenia (HR: 2.87; 95\% CI: 1.37 to 7.70; $p<0.002$ ), intra-aortic balloon pump (HR: $1.81 ; 95 \% \mathrm{CI}: 1.11$ to 1.86; $\mathrm{p}=0.021)$, and primary cardiac diagnosis (ischemic/other/unknown) $(\mathrm{p}=0.020)$.
\end{abstract}

Abbreviations: LVADs: Left Ventricular Assist Devices; CVAs: Cerebra Vascular Accidents; INR: International Ratio; CI: Confidence Interval

\section{Introduction}

Improving the survival and the quality of life of patients with end-stage heart failure has been the underlying goal since decades. Left ventricular assist devices (LVADs) have been increasingly important therapeutic option for the treatment of end-stage heart failure as 'bridge-to-transplantation'. In 2005 the American College of Cardiology/American Heart Association guidelines expanded the use of LVADs to include 'highly selected patients with refractory end-stage heart failure with estimated survival of $<50 \%$ at one year' [1]. Despite major advancements in medical management long-term use of LVAD comes with a toll of various adverse event like cerebrovascular accidents (CVAs), infections and bleeding that contribute to morbidity and mortality.

CVAs particularly hemorrhagic type though low in incidence continues to remain one of the leading mortalities associated with LVADs. There are several possible risk factors which may lead to hemorrhagic stroke in patients with LVAD, such as anticoagulation, hemorrhagic conversion after ischemic infarction, the acquired von Willebrand syndrome and rupture of fragile vessels. However, risk factors, characteristics and outcomes of stroke in patients with LVAD have not been well studied and there is less data in 
already published literatures. The goal of this study is to examine the various risk factors responsible for the hemorrhagic stroke and also to study the risk of mortality compared to non-stroke patients after LVAD placement.

\section{Materials and Methods}

We evaluated 2465 patients (mean age 61) who underwent implantation of HeartMate II (Thoratec Corp., Pleasanton, CA) or Heart Ware (Framingham, MA) at our center between March 2012 and July 2017. Stroke during LVAD support was analyzed. Competing outcomes analysis was used to determine which clinical risk factors were associated with the risk of stroke and death, with the primary end-point being time to first stroke event.

Diagnosis of stroke was made based on physical exam and supported by medical imaging such as a CT scan or MRI scan. Anticoagulation protocol included heparin, warfarin and antiplatelet agents except for those patients with contraindication to the medication or/and active bleeding.

Clinical characteristics, postoperative infection frequency, and anticoagulant status were compared between LVAD patients who developed stroke and those patients who didn't develop stroke. A postoperative infection was defined as symptoms of infection with concomitant positive microbiological cultures. LVAD related infection was defined as driveline, LVAD pocket, and/or wound and device infection. Urinary tract infection was defined as a single positive urine culture with $10 * 5$ colonies per milliliter with clinical signs of urinary tract infection, such as dysuria and/or pyuria.

Data were compared between groups using Student's 2-sample t-test for continuous variables and Fisher's exact test or Pearson's chi-square test for categorical variables. Non-normal and ordinal data were shown as median (first quartile, third quartile) and compared using the Mann-Whitney U test. A 2-sided p-value of 0.05 was considered significant.

\section{Results}

The mean patient age was 61 (SD, 7.5 years) with mean body mass index of 31 (SD, 5.9). The mean follow-up was 13.9 months (SD 9.45). During the study period, 2465 patients underwent continuous flow LVAD placement. Median follow-up was 9.79 months (range 0.02 to 34.96 months). Of all patients, 829 (33.63\%) had at least 1 stroke, with an incidence rate of 0.336 strokes per patient-year. A total of 356 (42.94\%) strokes were ischemic and 311 (37.522\%) were hemorrhagic. Patients with hemorrhagic stroke had worse survival than those with ischemic strokes (90day survival: $28.3 \%$ vs. $71.7 \%$; $p<0.001$ ). Of patients with a first stroke, $21 \%$ had a second stroke. Pre-implant predictors of stroke were female sex (hazard ratio [HR]: 1.25; 95\% Confidence Interval [CI]: 1.09 to 1.82; $\mathrm{p}<0.001$ ), pre-implant systolic blood pressure (HR: 1.87; 95\% CI: 1.00 to 1.01; p = 0.001), heparininduced thrombocytopenia (HR: 2.87; 95\% CI: 1.37 to $7.70 ; \mathrm{p}$ < 0.002), intra-aortic balloon pump (HR: 1.81; 95\% CI: 1.11 to 1.86; $\mathrm{p}=0.021$ ), and primary cardiac diagnosis (ischemic/other/ unknown) ( $\mathrm{p}=0.020)$. The risk of CVAs including hemorrhagic and ischemic two times more (HR: 1.67; 95\% Confidence Interval $[\mathrm{CI}]=1.64-5.35 ; \mathrm{p}=0.001]$ in patients with blood infection after LVAD placement. During a median follow-up time of 13.9 months, mortality risk in stroke patients was 2.01 times that of stroke-free patients (HR: 1.01; p: 0.002).

\section{Discussion}

Heart failure is one of the most common cause of death in the world. Each year up to 500,000 patients are diagnosed with HF with a total of 2,50,000 deaths in US. However, the number of donors remained static at approximately 2000 per year. This problem has been overcome by the recent advances and invention of left ventricular assist devices (LVAD). LVAD therapy has evolved into a standard therapy for patients with advanced heart failure, not only as a bridge to cardiac transplantation but also as a destination therapy [2].

Long term LVAD support, however, can result in serious complication, such as stroke, bleeding, thromboembolism, pump thrombosis, and infections. The incidence of stroke after LVAD replacement is reported to be $8 \%$ to $25 \%$. In another study, CFLVAD patients were monitored for both hemorrhagic and ischemic stroke, of them, 3\% developed ICH (intraparenchymal, subdural, and subarachnoid hemorrhage) subsequently. Although the risk of stroke is quite low compared to other complications such as bleeding; stroke, especially hemorrhagic type, is the leading cause of morbidity and mortality incapacitating some of the patients for heart transplant. It is the most common cause of the death after LVAD surgery. Several risk factors for stroke have been identified across studies which center on thrombus formation within LVAD itself, anticoagulation treatment, or infection [3].

Ischemic strokes may occur due to an embolus forming within LVAD or myocardium, though the correlation between clinical pump thrombosis and ischemic stroke appears less robust. Patients with embolic sources can develop ischemic strokes from different causes given their burden of cardiovascular disease risk factors, namely due to atrial fibrillation, artery to artery emboli from large artery atherosclerotic disease, or lacunar infarcts due to lipoylations of small cerebral arteries. Hemorrhagic strokes have been typically attributed to the anti-thrombotic treatment, though an elevated International Ratio (INR) is neither necessary nor sufficient to lead to hemorrhagic strokes. In clinical practice, patients may frequently have supra-therapeutic INR's without developing a hemorrhagic stroke, and conversely this stroke subtype may be seen in patients with subtherapeutic INR's. There are some risk factors associated with hemorrhagic stroke such as anticoagulation treatment, infection, high blood pressure.

In addition, a study separately analysed some of the risk factors for ischemic and hemorrhagic strokes. Gender, IABP use, hemoglobin, HIT and implant year, stand out as the important risk factors for hemorrhagic stroke. Risk of stroke with HeartMate 2 is higher in females compared to males, however the mortality remains the same in both. Another major risk factor for stroke 
in LVAD patients is high blood pressure which may cause stroke with several mechanisms; either directly by damaging to arteries (leading intracranial plaque formation, aneurysm formation and growth) or indirectly by causing cardioembolic events and extracranial atherosclerosis. MAP $>90 \mathrm{mmHg}$ before 7 days and including the day of accident was associated with a higher risk of hemorrhagic stroke [4].

People who develop ischemic infarct are at the risk of developing hemorrhagic infarct irrespective of their anticoagulation profile. Ischemic cerebrovascular accidents in patients with CF-LVAD are at higher risk for hemorrhagic transformation and this transformation is associated with larger infarcts and low platelet count at diagnosis. In addition, due to the biomaterials used in LVAD that activates coagulation system resulting pump thrombosis, patients need to use an anticoagulant such as heparin, warfarin, or direct thrombin inhibitor and one or more antiplatelet agents such as aspirin, clopidogrel, or dipyridamole, which causes diminished thrombotic profile in the patients and increases the risk of ICH [5].

Meanwhile, it's hard to reach optimal INR range in patients on warfarin, as individual warfarin dose affected by clinical conditions, individual's genetic variation, cytochrome p450 polymorphism and vitamin $\mathrm{K}$ epoxide reductase. In addition to chronic anticoagulant therapy, several other mechanisms are also hypothesized leading to bleeding after LVAD implantation such as acquired von Willebrand syndrome, reduce pulse pressure, and impaired platelet aggregation. Single centered studies have shown age, ischemic cardiomyopathy, hypertension, Body Mass Index (BMI), albumin, cardiopulmonary bypass time, and history gastrointestinal bleeding as the important pre-operative risk factors for bleeding after LVAD placement.

It is demonstrated that acquired von Willebrand syndrome occurs secondary to a large number of von Willebrand multimers cleavage after LVAD implantation that causes decreased platelet activity and aggregation. and in CF-LVAD patients, high shear stress alone causes degradation of large von Willebrand factor multimer into smaller multimers by physical abolition ,on the other hand , in presence of high shear stress ADAMTS-13 cleaves von Willebrand factor multimers into degradation products reducing the platelet accumulation and clot formation, so the therapy may be targeted towards ADAMTS-13 to decrease the cleavage of von Willebrand factor into smaller fragments decreasing the bleeding complications in patients with LVAD. All the patients on CF-LVAD are assumed to have a VWS and only small proportion of patients develop clinically significant bleeding due to a VWF which indicates bleeding in LVAD patients is a multifactorial process $[6,7]$. Although second generation-flow LVADs has overall good outcomes, however, device related infections are a frequent complication which causes significant financial burden because of increased hospitalization and surgical treatment.

Late device-related infections have become the main focus in patients whom LVAD is used for long term support or as a destination therapy. Device design, overall patient condition and immune dysfunction induced by LVAD increases the risk for developing device related infection. The mechanism by which LVAD can be a source for infection includes contamination of pathogens during implantation procedure, hematogenous spread of infection from distant site with secondary involvement of LVAD, entry of pathogens from the exit site in the skin. One way by which the susceptibility to infection in patients on LVAD therapy can be related is the defects in both cellular and humoral immunity which includes reduction in the number of CD4+T cells, increased apoptosis of T lymphocytes, defective macrophage response, B-cell hyperactivity leading to defective immunoglobulins productions which all predisposes the patients to widespread infection.

Moreover, there is proinflammatory cytokine imbalance in which there is decreases secretion of IL-2, TNF-alpha and increased IL-10 expression compare to patients who have advanced heart failure but without LVAD support. Device related infections can mainly be grouped into driveline infections, PumpPocket Infections (PPIs) and LVAD-associated endocarditis. Driveline infection along the percutaneous lead can ranges from erythema to fever, purulent discharge from the driveline exit and cellulitis. In addition, these patients are at increased risk for extension of this infection to the pump-pocket necessitating appropriate imaging to look for such involvement. Driveline infection is frequently associated with poor healing of the exit site. This problem especially becomes more important in patients on destination therapy as the duration of mechanical support is longer.

A study showed that driveline infections was not associated with increased incidence of thromboembolism, requiring the use of anticoagulation medication, however this risk was associated with septic emboli or rupture of mycotic aneurysm [8]. Pathogens commonly causing device related infection are Staphylococcus, Enterococcus and Pseudomonas species and in fact it is rare, some fungal species, especially candida can also cause device-related infection. Early LVADIs ( $<180$ days) are caused mainly by grampositive pathogens, commonly staphylococcus aureus, on the other hand, late LVADIs (>180 days) are caused frequently by gram negative pathogens, commonly by pseudomonas aeruginosa. In a study it has shown that pseudomonas aeruginosa Bloodstream Infection (BSI) is an important risk factor for CVA, especially for hemorrhagic subtype [9]. In the landmark REMATCH trial, 41 $\%$ of deaths were attributed to infections in patients on LVAD. In another study blood stream infection accounted for 7.9 per 1000 LVAD days in BTT patients and the main source of infection was device (38\%) and other sources included: catheters (16\%) respiratory infection $(6 \%)$, abdominal infection(6\%), urinary tract infection(1\%) and unknown (29\%), Mortality was highest for fungal infections, followed by gram negative bacilli and gram positive cocci .The inception of bloodstream infection after LVAD placement was longer when the microorganism was other than gram positive cocci [10-11]. 
Many studies failed to demonstrate an important relationship between demographic risk factors like age, gender or race with the development of driveline infection or PPI in pulsatile flow LVAD patients. Likewise, these factors do not seem to be associated with LVAD-related infections in continuous flow recipients. The risk of driveline infection arises when patients have metabolic derangements like diabetes. Similarly, when the cardiac function was improved through ventricular assist devices diabetes is brought into control and hence the risk for driveline infection and PPI decreases signifying the rate of risk for infection incidence in diabetics compared to non-diabetics. Raymond and his colleagues have shown that driveline infection is related with higher BMI proposing that obesity and associated metabolic syndrome predisposing LVAD patients also for device related infections [12].

Development of neurological complications after LVAD placement was separately related to history of cerebrovascular accidents and post-operative infection and it has been found that in patients supported with CF-LVAD, persistent BSI is more related to all subtype of CVA and mortality compared to other risk factors such as age, atrial fibrillation, high blood pressure [13]. Stroke after LVAD placement mainly involves right hemisphere which is more often associated with infection. Infection causes coagulations abnormalities that may give rise to thrombus formation resulting in right hemispheric stroke predominance [13]. We have a limited data about definition of infection, infection subgroups and infection origins in CF-LVAD patients and more importantly how infection leads to intracranial hemorrhage in these population is unclear.

\section{Conclusion}

Therefore, in our study, we focused more on hemorrhagic stroke and its association with infection. We noticed that postoperative infection increases the risk of stroke in LVAD patients that is associated with high risk morbidity and mortality. To reduce the risk of hemorrhagic stroke after LVAD implantation, it's essential to eliminate the risk factors, to optimize anticoagulant dose and to prevent infections in order to extend the survival benefit and reduce the mortality [14].

\section{References}

1. Hunt SA, Abraham WT, Chin MH, Feldman AM, Francis GS, et al. (2005) ACC/AHA 2005 guideline update for the diagnosis and management of chronic heart failure in the adult: A report of the American College of Cardiology/American Heart Association Task Force on Practice Guidelines (Writing Committee to Update the 2001 Guidelines for the Evaluation and Management of Heart Failure). J Am Coll Cardiol 112(12): e154-235.

2. Savarese G, Lund LH (2017) Global Public Health Burden of Heart Failure. Card Fail Rev 3(1): 7-11.

3. Christopher H, Laura H Ranjit J (2014) Left ventricular assist devices as a bridge to cardiac transplantation. J Thorac Dis 6(8): 1110-1119.

4. Frontera JA, Starling R, Cho SM, Nowacki AS, Uchino K, et al. (2017) Risk factors mortality, and timing of ischemic and hemorrhagic stroke with left ventricular assist devices. J Heart Lung Transplant 36(6): 673-683.

5. Willey JZ, Boehme AK, Castagna F, Yuzefpolskaya M, Garan AR (2016) Hypertension and Stroke in Patients with Left Ventricular Assist Devices (LVADs). Curr Hypertens Rep 18(2): 12.

6. Neal S, Parikh, Joséphine C, Maria GK, Amelia KB (2016) Stroke Risk and Mortality in Patients with Ventricular Assist Devices. Stroke 47(11): 2702-2706.

7. Sanjay M, Sreekanth K, Veli KT (2011) Left ventricular assist devicerelated infections: past, present and future, Expert Rev Med Devices 8(5): 627-634.

8. Trachtenberg BH, Cordero RAM, Aldeiri M, Molham A, Paulino A, et al. (2015) Persistent blood stream infection in patients supported with a continuous-flow left ventricular assist device is associated with an increased risk of cerebrovascular accidents. Journal of cardiac failure 21(2): 119-125.

9. Eric ARose, Annetine CG, Daniel FH, Lynne WS (2001) Long-Term Use of a Left Ventricular Assist Device for End-Stage Heart Failure. N Engl J Med 345: 1435-1443.

10. Gordon SM, Schmitt SK, Micah J, Nicolas MS, Marlene G, et al. (2001) Nosocomial bloodstream infections in patients with implantable left ventricular assist devices. Ann Thorac Surg 72(3): 725-730.

11. Raymond AL, Kfoury AG, Bishop CJ, Davis ES, Goebel KM, et al. (2010) Obesity and left ventricular assist device driveline exit site infection. ASAIO J 56(1): 57-60.

12. Aggarwal A, Gupta A, Kumar S, Baumblatt JA, Pauwaa S (2012) Are blood stream infections associated with an increased risk of hemorrhagic stroke in patients with a left ventricular assist device? ASAIO J58(5): 509-513.

13. Jeffrey JT, Mark SS, Joseph GR, Edwin CG, Francis DP (2015) The HVAD Left Ventricular Assist Device: Risk Factors for Neurological Events and Risk Mitigation Strategies. JACC: HEART FAILURE 3(10): 818-828.

14. Harvey L, Holley C, Roy SS, Eckman P, Cogswell R, et al. (2015) Stroke After Left Ventricular Assist Device Implantation: Outcomes in the Continuous-Flow Era. Ann Thorac Surg 100(2): 535-541. 

(C) Co This work is licensed under Creative DOI: $10.19080 /$ JOCCT.2018.12.555831
Your next submission with Juniper Publishers will reach you the below assets

- Quality Editorial service

- Swift Peer Review

- Reprints availability

- E-prints Service

- Manuscript Podcast for convenient understanding

- Global attainment for your research

- Manuscript accessibility in different formats

( Pdf, E-pub, Full Text, Audio)

- Unceasing customer service

Track the below URL for one-step submission https://juniperpublishers.com/online-submission.php 